\title{
Código guerrero: movilidad, guerra y muerte con lanzas
}

Patricio Trujillo Montalvo*

\section{Resumen}

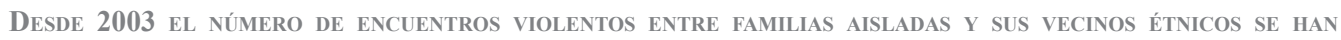
EVIDENCIADO CON MAYOR FRECUENCIA, LOS MISMOS QUE HAN PUESTO A LOS PRIMEROS EN COMPLEJOS ESCENARIOS DE VULNERABILIDAD. No EXISTEN ESTUdIOS PROFUNDOS, NI SISTEMÁTICOS SOBRE LOS PUEBLOS AISLADOS EN ECUADOR Y ESTE DESCONOCIMIENTO HA GENERAdo UNA SERIE DE ESPECULACIONES SOBRE ESTOS GRUPOS. El Mito deL No

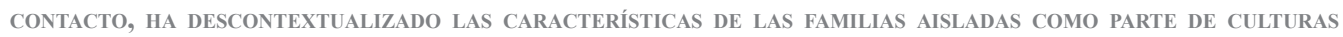
AMAZÓNICAS Y SU RELACIÓN DE INTERCAMBIO TANTO CON SU ENTORNO, COMO CON SUS VECINOS: FAMILIAS WAORANI, induStria petrolera, MADERERos, ETC. No EXISTE Grupos SIN CONTACTO, A LAS FAMILIAS AISLADAS, No SE LeS PODRÍA CATALOGAR COMO PUEBLOS OCULTOS, MENOS AÚN SIN CONTACTO, PUESTO QUE EXISTE EVIDENCIAS CIERTAS DE RELACIONES DE PARENTESCO Y ALIANZAS SIMBÓLICAS CON GRUPOS CLÁNICOS WAORANI CON QUIENES COMPARTEN UNA MISMA TERRITORIALIDAD.

Palabras claves: código guerrero - familias aisladas - grupos Clánicos - Movilidad - Culturas amazónicas - TERRITORIALIDAD.

\section{Abstract}

SINCE 2003 THE NUMBER OF VIOLENT CONFRONTATIONS BETWEEN ISOLATED FAMILIES AND THEIR ETHNIC NEIGHBORS HAVE OCCURRED MORE FREQUENTLY, WHICH HAVE PUT THE FORMER IN COMPLEX SITUATIONS OF VULNERABILITY. THERE ARE NO IN-DEPTH NOR SYSTEMATICS STUDIES REGARDING ISOLATED GROUPS IN ECUADOR AND THIS LACK OF UNDERSTANDING has GeNerated a SERIES OF SPECUlations ABOUt THEM. THE MYTH OF THE UNCONTACTED HaS DECONTEXTUALIZED THE CHARACTERISTICS OF ISOLATED FAMILIES AS PART OF AMAZON CULTURES AND THEIR INTERACTIVE RELATIONSHIP WITH THEIR ENVIRONMENT AS WELL AS THEIR NEIGHBORS: WAORANI FAMILIES, THE OIL INDUSTRY, LOGGERS, ETC. UNCONTACTED GROUPS DO NOT EXIST. THE ISOLATED FAMILIES CANNOT BE CONSIDERED HIDDEN GROUPS, MUCH LESS UNCONTACTED, OWING TO THE EXISTENCE OF CLEAR EVIDENCE OF FAMILY KINSHIP AND SYMBOLIC ALLIANCES WITH WAORANI CLAN GROUPS WITH WHOM THEY SHARE TERRITORY.

KEYWORDS: WARRIOR CODE - ISOLATED FAMILIES - CLAN GROUPS - MOBILITY - AMAZONIAN CULTURES - TERRITORIALITY.

* Doctor en Estudios Políticos por FLACSO, sede Ecuador; Master en Antropología Social por la Universidad de Estocolmo-Suecia; Licenciado en Antropología, PUCE. Director Científico de la Fundación de Investigaciones Andino Amazónicas (FIAAM). Profesor Asociado del programa de Estudios Interamericanos PUCE. Correo electrónico: 


\section{Introducción}

$\mathrm{E}$ ste ensayo, describe etapas históricas que conformaron la actual geografía de la región nororiental de la amazonia ecuatoriana y su relación con las familias indígenas aisladas. La industria petrolera, dinamizó la economía de esta región y se convirtió en el eje fundamental para el desarrollo del Estado, junto al dinamismo económico un fenómeno migratorio poblaba las selvas tropicales. La consolidación de poblaciones, en la amazonia ecuatoriana, está relacionada a un sistemático y acelerado proceso de urbanización. Es decir, a un nucleamiento sostenido, producto de la fisión de antiguas cooperativas y pre-cooperativas colonas campesinas, quienes ayudadas por la infraestructura vial construida por la industria petrolera, fueron construyendo, recintos, barrios, parroquias y ciudades. Toda esta compleja transformación social ha afectado y presionado de manera significativa a familias waorani y a familias de los "pueblos indígenas en aislamiento voluntario" (PIA) que es la forma oficial ${ }^{1}$ como se los designa y reconoce desde la normativa del Estado ecuatoriano. Es importante indicar, que los Waorani han aceptado la relación de intercambio social con la sociedad nacional, mientras que los grupos aislados, continúan dispersos, movilizándose en un territorio, cada vez más reducido y cercado.

Los resultados que se presentan en este ensayo son producto de años de investigación etnográfica ${ }^{2}$, que han priorizado entrevistas a guerreros waorani (Pikenanis ${ }^{3}$ ) que habitan en el Parque Nacional Yasuní (PNY) y la denominada zona Intangible Tagaeri-Taromenani (ZITT), quienes en su memoria oral, trasmiten un historial de encuentros e intercambio con familias Tagueiri y Taromenane, a quien la sociedad nacional denomina PIA, de diferente tipo como: a) enemigos-guerras, b) familiares- intercambios de alimentos, rituales y festivos.

La oralidad es fundamental para entender la cosmovisión del pueblo waorani. Las culturas orales, han desarrollado extensas y eficientes formas de comunicación simbólica. El mito es una de ellas y en palabras simples acapara, engloba, transmite, las sensaciones, sentimientos, la memoria de los pueblos mediante la utilización de cantos, leyendas, rituales, fiestas, testimonios, cuentos, relatos (Trujillo, 2011). La oralidad, para los Waorani está relacionada a la guerra y a como esta construyó su cotidianidad, el día a día de los diferentes clanes, como configuró el ambiente y a quienes lo rodeaban (las fronteras, el paisaje), como imaginaron un espacio-territorio alrededor de una ecología inagotable en recursos y de enemigos a quien había que eliminar para vivir en paz. Complementario a la oralidad, es fundamental entender, las complejas relaciones de parentesco y formas de designación social y reconocimiento con otros grupos waorani (waranis) y con sus vecinos étnicos, considerados enemigos o cuwuri (Ibíd., 2001, 2003, 2011).

\section{Movilidad y territorialidad}

Rivas (2014), considera que los $\mathrm{PIA}^{4}$ constituyen los últimos grupos de hombres y mujeres de la Amazonía ecuatoriana y mundial, que no permanecen en contacto estrecho con la sociedad

1 Plan de Medidas Cautelares, del Ministerio de Justicia de la República del Ecuador (2007).

2 Se utilizaron técnicas etnográficas como: RAP (Rapid Anthropolological Procedeures) y REA (Rapid Ethnographical Procedures), que se concentran en temas específicos y con objetivos concretos, en este caso la cosmovisión sobre: historia del pueblo, significado de la guerra, relaciones de parentesco, movilidad, grupos amigos, aliados (Waranis) y enemigos (Cuwuris), grupos Tagueiris, grupos Taromenane y otros potenciales grupos que viven en la selva en forma tradicional según la cosmovisión Waorani (Durani bai). Las entrevistas fueron realizadas con el apoyo de un investigador waorani que colaboró como intérprete y a la vez como informante. Se utilizaron preguntas abiertas con la finalidad de recopilar información sobre: a) Historia-biografía del entrevistado (nombre y procedencia-lugar, infancia, relaciones familiares, padre-madre-parentesco b) Guerras y paz, conocimiento sobre los Tagueiri y los Taromenane y otros grupos aislados c) Geografía de guerras: Imaginarios sobre la guerra, conflictos con otros grupos, futuro de los Waorani. Con la información obtenida se reconstruye una parte de la historia de estos pueblos, sus espacios simbólicos y la geografía de la guerra en relación con los grupos en aislamiento.

3 Designación del wao terero al guerrero y anciano Waorani.

4 Sobre los PIA se tiene información fragmentada, poco sistematizada y sin evidencias ciertas. Esto ha provocado un sin número de especulaciones sobre estos grupos, su localización, formas de reproducción social, pertenencia étnica y sobre todo sobre su relacionamiento con sus vecinos. 
envolvente, ya sea por una decisión propia conforme sus normas culturales, o por su permanente huida frente a sus enemigos y a los efectos que la civilización causa en sus vidas y culturas. En el Ecuador, las familias aisladas, Tagueiri-Taromenane, tienen pertenencia lingüística, wao terero $^{6}$, adicional a una forma de movilidad parental uroxilocal ${ }^{7}$ (Narváez, 2016). Los PIA, en su relación con sus parientes culturales, los waorani, atraviesan por dimensiones tanto simbólicas y territoriales, conformando un complejo sistema de competencia por recursos, puesto que se movilizan en espacios conjuntos, a esta relación se debe sumar componentes simbólicos, relacionados a la guerra, donde una serie de episodios de matanzas y venganzas han configurado una compleja geografía bélica entre estos dos grupos (Trujillo, 2016).

La situación de los PIA, en Ecuador, es compleja, puesto que se encuentran presionados por la expansión de la frontera agrícola, por un uso compartido y conflictivo del mismo territorio de caza y recolección por familias waorani, shuar, kichwa y colonos-campesinos; además de turistas $^{8}$ que recorren ríos y zonas cercanas a sus lugares de movilidad; y, finalmente por las actividades de extracción, principalmente petroleras, mismas que se han ampliado hacia la zona de influencia de estos grupos como es el caso de los pozos hormigueros y el desarrollo del campo marginal Armadillo'.

\begin{abstract}
"Pegonta, vive en Miwaguno, un asentamiento Waorani, que se consolida por la presencia de la empresa petrolera, las vías y todo el sistema clientelar que estas tiene con la población Waorani. Le encuentro afilando su lanza, dice que sus nietos le roban para vender en las ciudades y que no tiene con que ir de cacería, no es matar gente me dice, eso ya no lo hacemos, es para cazar, en esta época, marzo están gordos los ure (puercos de monte) de tanto comer chonta, yo salgo a cazar traigo comida para mi familia. Pegonta nos relata sus encuentros con familias aisladas, insiste que son familias, que se reconocen, él cuando sale al bosque va desnudo para que lo reconozcan, con sus lanzas para cazar y su escopeta para defenderse de enemigos. Relata que puede caminar en menos de un día hasta la zona de Wane en Ñoneno, cerca de los pozos Armadillo, conoce los caminos por donde sus padres y abuelos caminaban, esos son los mismos caminos que usan los Tagueiri, ellos son rápidos para moverse, llegan aquí entre enero y marzo, ahí donde van hay mucha chonta sembrada, tienen mucha caza, hacen fiesta, vienen silban como pajaritos, dejan carne de ure (cerdo de monte) se llevan un poco de yuca y plátano, yo les dejo, se van y regresan, a veces llegan en la noche, preguntan si conozco a Davo, que él mato a sus familiares, que lo están buscando, luego se van" (Diario de campo, 2013).
\end{abstract}

5 Brasil registra varios pueblos en aislamiento entre los que se destacan los korubo, hi-merima, massaco, zo'e, pipiticua, awá, caru, araribóia, kampa, menkragnoti, machineri, jaminawa, maku-nadeb, akurio, jandiatuba, piriuititi, jamamadi, familias kayapó pu ró, tupi, waiapiianeana, a más de otros. En Perú se registran los yine, yora, pano, cashibo-cacatiabo, familias matsiguenga, yora y ashaninka, sharanahua, yaminahua, chiltonahua, cuñarejo, mashcopiro-iñapari, kugapakori, nahua, murunahua, iconahuaa. En Bolivia cinco grupos étnicos: taromona, nahua, mbya-yuki, ayoreo y pacahuara. Colombia registra un pueblo en aislamiento en la región del río Puré, los yuri o caraballo. En Paraguay se registran varias familias del grupo étnico ayoreo quienes ejercitan su movilidad en regiones fronterizas con Bolivia. Ecuador cuenta con al menos dos pueblos indígenas en aislamiento vinculados a la nacionalidad Waorani, estos pueblos son los Tagaeri y Taromenane, no se debe descartar la existencia de otras familias y clanes aún desconocidos (Rivas, 2003).

6 Wao terero o tiriro, es la forma de denominar a la lengua waorani.

7 El grupo se moviliza en territorio, que en la memoria oral es de las mujeres, es decir las descendientes de Tague.

8 Existe una operadora de turismo "Shiripuno Lodge" que se encuentra en el borde del área de la ZITT con una notable afluencia de turistas. En convenio con la familia de Wane quienes reciben beneficios económicos de esta actividad, los turistas pasean por el bosque acampan en las playas del Shiripuno y conviven con miembros de familias waorani que sirven de guías e informantes. Existe también evidencia de un tipo de turismo que oferta la posibilidad de "encuentros" con grupos aislados, de menor afluencia, que es ofertado por miembros de las familias de Baameno y Boanamo. Este tipo de turismo es de alto impacto para los grupos aislados puesto que a decir de nuestros informantes: espantan la cacería, acampan en las playas que en época de huevos de charapas son visitadas por los grupos aislados y sobre todo la presencia de extraños molesta a los grupos aislados que vigilan la parte media, baja del río Shiripuno y la confluencia con el río Tiwino.

9 La explotación petrolera facilita la construcción de vías que a la vez facilitan asentamientos permanentes de grupos; colonos-campesinos (Los Reyes), Waorani (Miwaguno y Yawenpae, Noneno) y shuar (Tiwano), quienes presionan cada vez más los territorios por donde se movilizan los grupos aislados en especial los localizados entre el área Tivacuno-Armadillo-Cononaco Chico-Menkaro. 
El modelo de territorialidad y movilidad de los PIA, se encuentra atravesado por dos variables (Trujillo, 2013, 2014): a) tiempo y b) espacio. El tiempo, se refiere a la cada vez más cercana frecuencia (años, meses) de encuentros entre familias aisladas y sus vecinos étnicos: Waorani, colonos, madereros, etc.; mismos que generalmente han sido violentos y que han causado muertes de lado y lado. Espacio se relaciona a la territorialidad de los pueblos indígenas aislados, vinculada a su forma de uso tanto de recursos como de espacialidad. El espacio se reduce por la expansión de frontera agrícola, la presencia de centros urbanos e industrias (petrolera, turismo, maderera), provocando la reducción de los espacios vitales de movilidad de los PIA relacionados: caza, pesca y recolección. A la vez la reducción del territorio provoca espacios de competencia por recursos con Waorani cercanos (principalmente animales de cacería: monos, puercos saínos, tapires, pavas de monte, otros).

\section{TIEMPO}

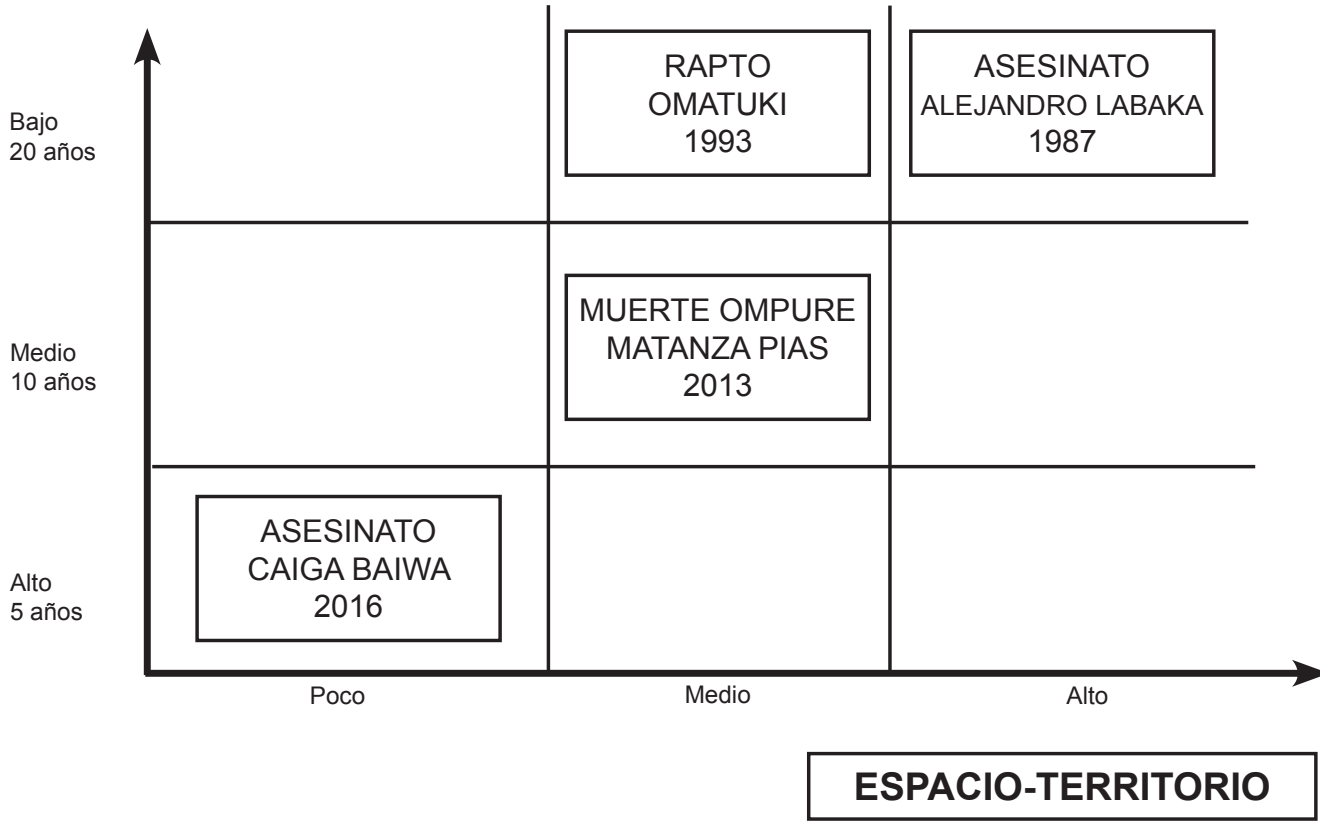

FigURA 1. Modelo de tiempo y espacio. Elaboración del autor, 2016.

Este modelo de territorialidad propuesto, sugiere que mientras menor sea el espacio para la movilidad (patrón de reproducción sociocultural de los PIA), mayor será en el tiempo, la posibilidad de encuentros con sus vecinos, lo que implica una mayor posibilidad de eventos violentos que amenacen y vulneren su existencia. Al contrario, mientras mayor sea el espacio de movilidad y reproducción de los PIA y sus patrones culturales, será menor la posibilidad de encuentros con sus vecinos y por ende menor la cantidad de eventos violentos. 


\section{MENOR ESPACIO \\ TERRITORIAL}

\section{MAYOR POSIBILIDAD \\ DE ENCUENTROS \\ VIOLENTOS}

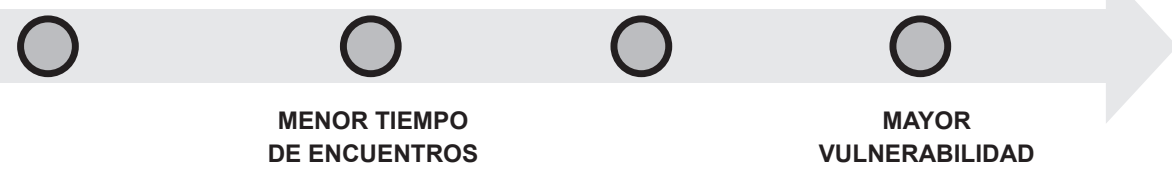

FigURA 2. Modelo Uno, tiempo-espacio-vulnerabilidad. Elaboración del autor, 2016.

\section{MAYOR ESPACIO \\ TERRITORIAL}

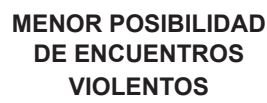

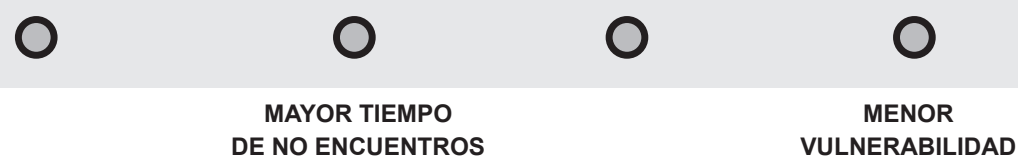

FigURA 3. Modelo Dos, tiempo-espacio-vulnerabilidad. Elaboración del autor, 2016.

Es evidente que la ampliación de la infraestructura petrolera, las carreteras, así como la expansión de la frontera agrícola, colaboran en la presión a la fauna que habita el sector, base fundamental de la dieta de las familias aisladas ${ }^{10}$. Es importante entender que la territorialidad de estas familias se basa en su amplia movilidad y en la relación con recursos que pueden obtener de la selva, así los sitios de recolección, de cacería, de pesca, son fundamentales para delimitar lo que imaginan y definen como su espacialidad-territorio.

\section{Carreteras, los accesos a la civilización y contacto}

La colonización, en la región amazónica fue mayoritariamente espontánea, es decir, que los colonos campesinos se fueron posesionando de zonas boscosas, formando pre cooperativas y luego legalizando sus tierras ${ }^{11}$, lo que provocó múltiples conflictos con áreas de reserva e indígenas, a más de tala ilegal y venta de madera ${ }^{12}$. La explotación de recursos, se convirtió en la principal propuesta de desarrollo impulsada desde el Estado (Trujillo, 2001), enmarcado como un proyecto nacionalista, que fue promovido por la dictadura militar (1972-1979), donde se imaginó a la amazonia como el gran abastecedor de riquezas para todo el país ${ }^{13}$. La implementación del proyecto modernizador, significó que la economía nacional

10 En el informe de FLACSO-Pachamama, 2012, que tomó como referencia los mismos informantes se resalta que: “su base alimenticia está en las presas de caza y prefieren los monos, las guanganas, los paujiles y pavas de monte. Así también harían algo de pesca pero usando lanzas y palos. Del bosque recogerían plantas medicinales y alimenticias, entre ellas las hojas de ajo, pepas, frutas, tapemo, ungurahua, uva de montes.

11 Existió intentos de ordenamiento de los puntos de colonización, a través de programas de colonización dirigida y semi-dirigida, que no tuvieron el éxito esperado: Shushufindi (1972), Payamino (1976), San Miguel (1977), CAME 3 y Loreto-Coca (a cargo del IERAC), fueron los proyectos pilotos de colonización, sin embrago estos no pudieron auto sostenerse y la falta de dinero los desarticuló (Trujillo, 2001).

12 La apertura de vías facilitan el transporte de madera, en especial en las vías que conducen a pozos petroleros, la presión hacia el bosque ha generado encuentros violentos y lanceamientos a colonos por miembros de los grupos aislados que cada vez se encuentran más presionados por la agresiva apertura de la frontera agrícola.

13 Años antes a esta etapa histórica, nuestro país centraba su economía en la producción agrícola local (Larrea, 2008). 
se basaría en la explotación de recursos naturales a gran escala (petróleo y minerales), que a la postre fue la principal estrategia del Estado para integrar la región con la sociedad nacional.

Esta forma de construir a la amazonia y a sus habitantes, legitimó desde el Estado prácticas de intervención, delineando nuevas definiciones sobre la planificación, sobre el espacio y la estética de la población. Las misiones cristianas (católicas o evangélicas) y las empresas petroleras, se convirtieron en los ejes de la integración espacial y social de las poblaciones hacia la "sociedad mayor", integrándola hacia la modernidad y una visión de desarrollo, que promovía formas de manejo de la naturaleza y ocupación territorial con una visión civilizatoria occidental (Ibíd., 2011).

\section{La famosa vía Auca}

En los últimos 20 años, en las provincias amazónicas, se evidencia un rápido proceso de urbanización. La matriz primigenia que fueron las pre-cooperativa y cooperativas rurales colonas campesinas, ya no existen, dando origen a pequeños asentamientos urbanos, que se vinculan ahora a ciudades y a nuevas formas de organización política, como parroquias, cantones y provincias $^{14}$.

Como vía Auca, se conoce a un complejo vial construido por la empresa petrolera Texaco, que inicio en 1970 y culminó en 1980 del siglo pasado, con más de $120 \mathrm{~km}$. de carretera desde la ciudad de Coca hasta la comunidad waorani de Tiwino-Bataboro. La infraestructura petrolera realizada por Texaco desplazó bruscamente a familias waorani del grupo Niwairi, quienes tenían su territorialidad dentro de toda esta zona. El contacto fue violento y provocó constantes enfrentamientos entre militares, empleados de las compañías petroleras, guerreros waorani (Ibíd., 2011). La vía dividió al territorio tradicional waorani, ocasionando que familias de este grupo étnico, quienes usaban esta región como su espacio de movilidad y reproducción sociocultural, migren hacia el sur-este, provocando conflictos por límites territoriales y sobre todo por competencia de recursos como la cacería ${ }^{15}$.

"La ciudad de Coca creció como un polo de desarrollo petrolero, desde allí hacia el sur-este sale una vía carrozable que se denomina como Auca, es una larga carretera de más de 120 kilómetros que parte el territorio Waorani. Al viajar por esta vía se puede observar el proceso colonizador que se desarrolló desde los años de 1970 con el apoyo del Estado, con la ley de colonización, tierras baldías, en estrecha relación con las empresas petroleras. Texaco fue la compañía que desarrollo los pozos Cononaco y Auca, mismos que al entrar en producción permitieron el desarrollo industrial petrolero de la zona, la construcción de vías de acceso y el aparecimiento de poblados tanto de grupos colonos mestizos que llegaban en búsqueda de tierras para producir (ganado, café) y de otros grupos indígenas amazónicos (Kichwa y Shuar). A casi 40 kilómetros del Coca, se encuentra el poblado de Dayuma, con un crecimiento desordenado y caótico demuestra como la matriz primigenia que fue la pre-cooperativa rural o la comuna cambió a asentamientos nucleados a lo largo de las múltiples vías en el sector, razón por la cual muchas de las antiguas pre-cooperativas y cooperativas colonas ya no existen dando origen a pequeños asentamientos que han provocado un rápido proceso de urbanización con la creación de diferentes barrios, recintos, comunas, parroquias, cantones y ciudades. Siguiendo unos kilómetros más por la vía hacia pozos Pindo se encuentra

14 La rápida urbanización ha convertido los antiguos poblados en los centros de acopio comercial, las nuevas poblaciones forman barrios, utilizando la infraestructura de comunicación vial. Estos barrios evidentemente empiezan a solicitar otras demandas y se expresan en otras formas de organización. Los servicios básicos como: agua potable y alcantarillado se convierten en las principales necesidades que deben ser satisfechas para mejorar las condiciones de vida; así, como también en lo referente a los servicios de educación y salud.

15 Entre las décadas de 1990-2000, una de las principales característica de la sub región petrolera fueron los constantes brotes de violencia y conflictos que enfrentaron a poblaciones campesinas-colonas, indígenas y militares, muchos de los cuales fueron mediados por la Iglesia Católica. Los conflictos por tierra fueron los principales escenarios de violencia y tensión en la sub región petrolera (Trujillo, 2001; 2014). 


\begin{abstract}
Davo, otro guerrero Waorani, hijo de Niwa, quien fuera el que controlará toda esta región. Davo y su esposa Zoila viven en la $\mathrm{Y}$ de Pindo, tienen una casa de fabricación mixta y una gran cadena que la tensan cuando llega un auto o un camión, deben pagar peaje para pasar por su territorio me dice Zoila, quien habla castellano. El pago del peaje puede ser en dinero, pero también en comida y frecuentemente en coca cola. Más adelante sigue la sinuosa carretera construida por Texaco, misma que fue el inicio de la penetración de colonos campesinos que fueron asentándose conforme las vías van a pozos y a estaciones, campos de café, pastos y muchas pequeñas pre cooperativas y cooperativas que da una nueva forma a la región amazónica. Este fue el territorio de mi papa, me dice Davo, Ñiwa el mejor guerrero Waorani, el defendía su territorio de los cuwuris, los extraños, tenía una casa grade en lo que es ahora el campamento de Cononaco, ahí nací yo. La carretera finaliza en la zona denominada Tiwino que es el inicio del territorio Waorani, allí una cadena divide los dos mundos, el Waorani y el colono. En la cadena esta Babe, alto y fornido, junto a una botella de coca-cola cobra peaje a los gringos turistas que van río abajo a las cabañas de la compañía turística, siguiendo la vía existen aún 20 kilómetros que han sido tapados por la selva, fueron construido por Petro-Canada, la empresa que transporto a Babe y a toda su familia hacia este sitio denominado Tiwino, el proyecto fue llegar hasta el río Curaray y así completar toda una gran zona petrolera, no lo pudieron hacer, según me relatan es tierra donde viven indios bravos, les dicen los pata colorada, o los Tagueiri, ellos no permiten el paso, mata con lanzas a los que caminan por este lugar" (Diario de campo, 2009).
\end{abstract}

La apertura de vías, permitió una rápida entrada de colonos campesinos, quienes fueron posesionándose de los predios junto a los campamentos y carreteras ${ }^{16}$. Los colonos campesinos provenientes de Loja, Bolívar, Manabí, Pichincha y El Oro, así como grupos familiares de la nacionalidad Kichwa y Shuar, se asentaron preferentemente en zonas cercanas a las riberas del río Napo y el amplio complejo de vías carrosables, construidas por las empresas petroleras. De esta forma, ingresaron a la vía Auca, zona declarada por el gobierno nacional como tierras baldías, y por ende para uso agrícola ${ }^{17}$ y rápidamente desplazaron a sus antiguos habitantes. El agresivo desmonte de la selva se origina fundamentalmente por las mismas leyes que regían la colonización, y que pedían un trabajo de más del $50 \%$ del predio para poder ser adjudicado ${ }^{18}$ (Ibíd., 2001). La carretera, fue el principal ingreso hacia zonas del Parque Nacional Yasuni (PNY), territorio étnico Waorani y la Zona Intangible Tagaeri-Taromenane (ZITT). En la actualidad es un verdadero complejo regional, alrededor de la infraestructura petrolera, que combina una agresiva apertura de la frontera agrícola, urbanización sin planificación y desarrollo de la industria petrolera.

\title{
La vía Maxus
}

La denominada vía Maxus, es una carretera que fue construida en la década de los 90, del siglo pasado, cuando inicio la apertura de los campos de producción petrolera, hacía zonas más orientales del Parque Nacional Yasuní. La vía, conforma un complejo sistema industrial, de 131,5 kilómetros, desde el denominado Puerto Pompeya hasta los pozos Iro. El primer tramo de 42,5 kilómetros, sirvió para llegar a los campamentos cerca de los campos Capirón,

16 Los primeros asentamientos de los colonos campesinos, se construyeron alrededor de los campamentos y la infraestructura petrolera como por ejemplo Taracoa, Dayuma, Cononaco, Tiwino.

17 "Las normas legales que tienen relación con el acceso y uso del suelo son: la ley de Tierras Baldías y Colonización publicada en el registro oficial N. 342 del 28 de Octubre de 1964; el Reglamento para la adjudicación de tierras Baldías, publicado en el registro Oficial N.253 del 25 de Junio de 1971; la ley de Colonización de la Región Amazónica Ecuatoriana publicada en el Registro Oficial N.504 del 12 de Enero de 1978 y la Ley Forestal y de Conservación de Áreas Naturales y Vida Silvestre. Las Instituciones a las que las normas legales confieren competencia para la aplicación son: El Ministerio de Agricultura y Ganadería, el Instituto de Reforma Agraria y Colonización y el Instituto Nacional de Colonización de la Región Amazónica."

18 Trabajar este 50\% significaba haber botado grandes extensiones de bosque primario. Las graves consecuencias de las mismas leyes y del rápido proceso colonizador determino que en pocos años esta zona se encuentre casi totalmente deforestada. 
Tivacuno y Bogi, donde se hicieron ramales de la carretera $(13 \mathrm{Km}$.) para sus accesos. La vía fue denominada como "carretera ecológica", y durante los casi 10 años de su construcción, fue uno de los principales conflictos entre Estado, compañía petrolera y organizaciones ecologistas-ambientalistas, quienes advertían un alto impacto sobre el Parque Nacional Yasuní y la población waorani (Ibíd., 2001).

"El río Napo, es la principal vía fluvial, conduce hacia el río Amazonas. Desde la ciudad de Coca son casi 4 horas en lancha rápida hacia Puerto Pompeya, es el sitio de ingreso hacia el bloque petrolero de Maxus, conocido como bloque 16. Navegar río abajo por el Napo, es una experiencia única, el río da vueltas e inunda grandes zonas de bosque, cruzamos varios poblados antiguas haciendas de la época cauchera que aún conservan los nombres. El ingreso a Puerto Pompeya es similar al ingreso a una embajada extranjera, puestos de control, revisión de papeles, permisos, requisas. El ingreso al bloque 16 se convierte en una zona de seguridad donde el Estado ecuatoriano, aparentemente perdió soberanía y las reglas de la petrolera rigen desde que pasamos los controles y subimos al bus. El recorrido por la famosa vía ecológica se hace bajos estrictos controles de velocidad, no se puede rebasar los 40 kilómetros por hora, el paisaje es bosque y chacras de pobladores Kichwas quienes aprovechando la etapa de colonización, promovida por el Estado entre la década de los 80 fueron posicionándose de las riberas del río y luego declarando como suyo parte de lo que fue el territorio ancestral Waorani. Hasta el kilómetro 32 es territorio de la comuna Kichwa de Pompey e Indillama. La frontera que divide a Kichwas y Waorani es el río Tiputuni, Giuyero para los Waos, ahí se encuentra el primer asentamiento permanente de los Waorani formado por la familia de Kawena Awa, un viejo Waorani, del que me dicen es cazador no guerrero, por lo que es tranquilo, sus hijos Tigue y Mingui viven cerca al igual que Nambai, que mantiene lazos de parentesco, pero él es hijo de Iteka, un emblemático guerrero Waorani. Cerca al kilómetro 42 está el campamento denominado NPF (North Production Facilities) de la compañía Maxus, que posteriormente venderá sus acciones al consorcio petrolero ArgentinoEspañol Repsol-YPF. Las facilidades petroleras han provocado una sistemática migración de familias Waorani que se han asentado a lo largo del campamento, allí obtiene comida, es como su nueva fuente de provisión, el cazador espera entre las rejas de los campamentos para obtener su sustento diario" (Diario de campo, 2005).

Luego de más de 20 años de explotación petrolera en esta zona, se considera que el mayor impacto producido por la carretera, ha sido la presencia de asentamientos permanentes ${ }^{19}$, lo que cambio los patrones de movilidad de familias Waorani, provocando, a la vez, una sobreexplotación de los suelos selváticos, y una presión más sistemática hacia zonas de movilidad de familias aisladas (Trujillo, 2011). Los accesos de la carretera hacia el mundo de afuera, mercados, campamentos petroleros, se convirtieron en un espacio atractivo para los Waorani, quienes apadrinados por la petrolera recibían recursos monetarios, convirtiéndose en trabajadores de la empresa ${ }^{20}$.

\section{¿Quiénes son las familias aisladas?}

Para Davo y Pegonta, existen por lo menos tres grupos o familias aisladas que se mueven entre las cabeceras del Tivacuno (Peeneno), Shiripuno (Keweriono), Cuchiyaku (Tiwino), Nashiño (Gabaron) y Yasuní (Kawymeno). La primera, estaría conformada por "descendientes o supervivientes" del grupo familiar de Tague ${ }^{21}$, que al parecer fueron absorbidos por familias del

19 Los nucleamientos provocan, exigencias de infraestructura básica como: casa comunal, escuela, puesto de atención médica. Con los nucleamientos se empieza un rápido deterioro en la calidad de vida, disminuye la caza, y empieza un rápido aparecimiento de enfermedades tropicales como leshmaniasis y malaria.

20 Es un polo de atracción, por las oportunidades de empleo, por la facilidad de movilización, por atención médica que brinda la empresa a los miembros de ésta etnia, por la posibilidad de aprovisionarse de víveres.

21 Tague fue asesinado, en 1986 cuando una barca de la Compañía General de Geofísica (CGG) se dirigía hacia el campamento base de sísmica localizado en la unión de los ríos Shiripuno y Cononaco Chico, zona que constantemente era atacada por guerreros de este grupo (Trujillo, 2011). 
grupo denominado como Taromenga-Taromenani ${ }^{22}$; estas familias ${ }^{23}$ se caracterizan por una alta movilidad vinculada a épocas de cosecha (frutos como chonta, uncurahua, uvas), recolección (huevos de charapa, gusanos de chonta) y cacería (monos).

"Llegan a los lugares donde los abuelos sembraron chonta entre los meses que dan los frutos (febrero-mayo), tiempo bueno para la cacería, los monos están gordos, yuca también tienen, pero cuando se acaba vienen a conseguir por aquí cerca de nuestras chacras, a veces dejan carne de guangana "ure" en canastas... silban como pájaros y se van llevando yucas o plátanos"24.

"Desde el kilómetro 58 de la vía Maxus hasta el 80 es territorio, Tagueiri" Pego es un guerrero waorani, es hijo de Wane, hermano de Iniwa, Kempery y Yety. Vive en Peeneno, con Tamata hija de Awañete, quien fuera esposa de Yety y Pego en una relación de poliandria. La poligamia en sus dos formas, hombres con varias mujeres (poligamia) y mujeres con varios hombres (poliandria) es reconocida y aceptada socialmente entre familias de varios clanes waorani. La casa (onko) de Pego está localizada en el kilómetro 58 de la vía conocida como Maxus, misma que conduce a los pozos petroleros de la empresa Repsol-YPF. Habla muy poco castellano, pero es muy expresivo, nos muestra la tumba de su hermano Yety, cerca de su casa, y relata que lo mato, que debía hacerlo, caso contrario nunca tendría una mujer, una vez muerto Yety, Pego tomó a Tamata como su mujer, ella solo nos mira y sonríe. Pego conoce a los Tagaeri, sabe dónde viven y nos relata que frecuentemente va a visitarlos y a retarlos con su lanza, su deseo es matar Tagaeris, son sus enemigos. Pego no sabe dibujar, sus trazos son líneas en varias direcciones, dirige oralmente a sus sobrinos la geografía de su territorio, y estos hábilmente lo plasman en un dibujo (ver ilustración 1). El mapa de Peneno es un complejo geográfico donde se visibiliza la carretera de Maxus, el poblado de Diicaro los pozos petroleros Amo, las zonas de cacería, la casa de Pego y Tamata, la tumba de Yety, el río Peeneno (Tivacuno) y en cabecera del mismo una casa Tagueiri. Siguiendo la carretera, desde el kilómetro 58 hasta el 80 no existe nada más que zonas de bosque, taromes o áreas de cacería, no hay más asentamientos Waorani, puesto que es territorio de los Tagueiri" (Diario de campo, septiembre 2000).

Estas familias PIA, según la información sistematizada, serían el grupo más vulnerable y presionado: a) por los grupos Waorani vecinos, b) por la explotación ilegal de madera, c) por la expansión de la frontera agrícola; $y, d$ ) por la expansión de la actividad petrolera, puesto que se moviliza entre la cabecera del río Tivacuno, el río Cononaco Chico y Menkaro. Son además, las que mayores registros de contactos violentos y ataques por parte de guerreros waorani, han tenido con consecuencias fatales ${ }^{25}(1993,2003,2013)$. De acuerdo a los informantes entrevistados, se encontrarían debilitados y en grave riesgo de desaparecer.

22 Los Taromemani, es una palabra Waorani que sirve para designar a todos quienes viven en el bosque (omaere durani bay), su traducción literal del wao terero al español es la "gente del camino o del monte". La palabra "tarome-na" se usa para designar los caminos de cacería que todo guerrero tiene y controla como su área de acción. Taromenani, también es la palabra para designar a familias aisladas con poco contacto, que mantienen una alta movilidad entre los ríos Nashiño y Cononaco.

23 El Plan de Medidas Cautelares/Ministerio de Justicia Derechos Humanos y Cultos ha recolectado información en la zona que demuestra la presencia de los PIA, entre las evidencias se encuentran: fotos de pisadas y casas abandonadas, lanzas que en su forma y decoración no pertenecen a ninguna familia Waorani conocida.

24 Relato de Pegonta, Miwawuno, agosto 2013.

25 La investigación de FLACSO y la fundación Pachamama (2012) revela interesantes datos respecto a los enfrentamientos en la zona de estudio: "haciendo un recuento histórico de ataques del grupo Tagueiri - Taromenane en la zona cercana al campo Armadillo se tiene que en 1971 ocurre un enfrentamiento contra trabajadores de la compañía Western; luego en 1977 mueren lanceados tres trabajadores de la CGG y muy próximo al río Shiripuno en 1987 muere monseñor Alejandro Labaka e Inés Arango. En el mismo año mueren cuatro personas más, esto incluye a dos personas Tageiri, en 1993 en el sector de Cuchiyaku fallece Carlos Omene y para 1993 el grupo de Babe secuestra a Omatuki. Posteriormente, en el 2002 mueren cinco madereros muy cerca de Noneno, en el 2003 mueren 16 personas del clan Tageiri-Taromenane. Como hechos recientes, entre el 2007 y 2008 se dieron varias muertes de madereros, entre ellos Mariano Castellanos; en el 2009 mueren lanceados tres colonos en Los Reyes; el 4 de abril de 2014 se registra un nuevo enfrentamiento con la muerte por lo menos de dos trabajadores estatales de Ecuador Estratégico en Tigüino-Bataboro. 


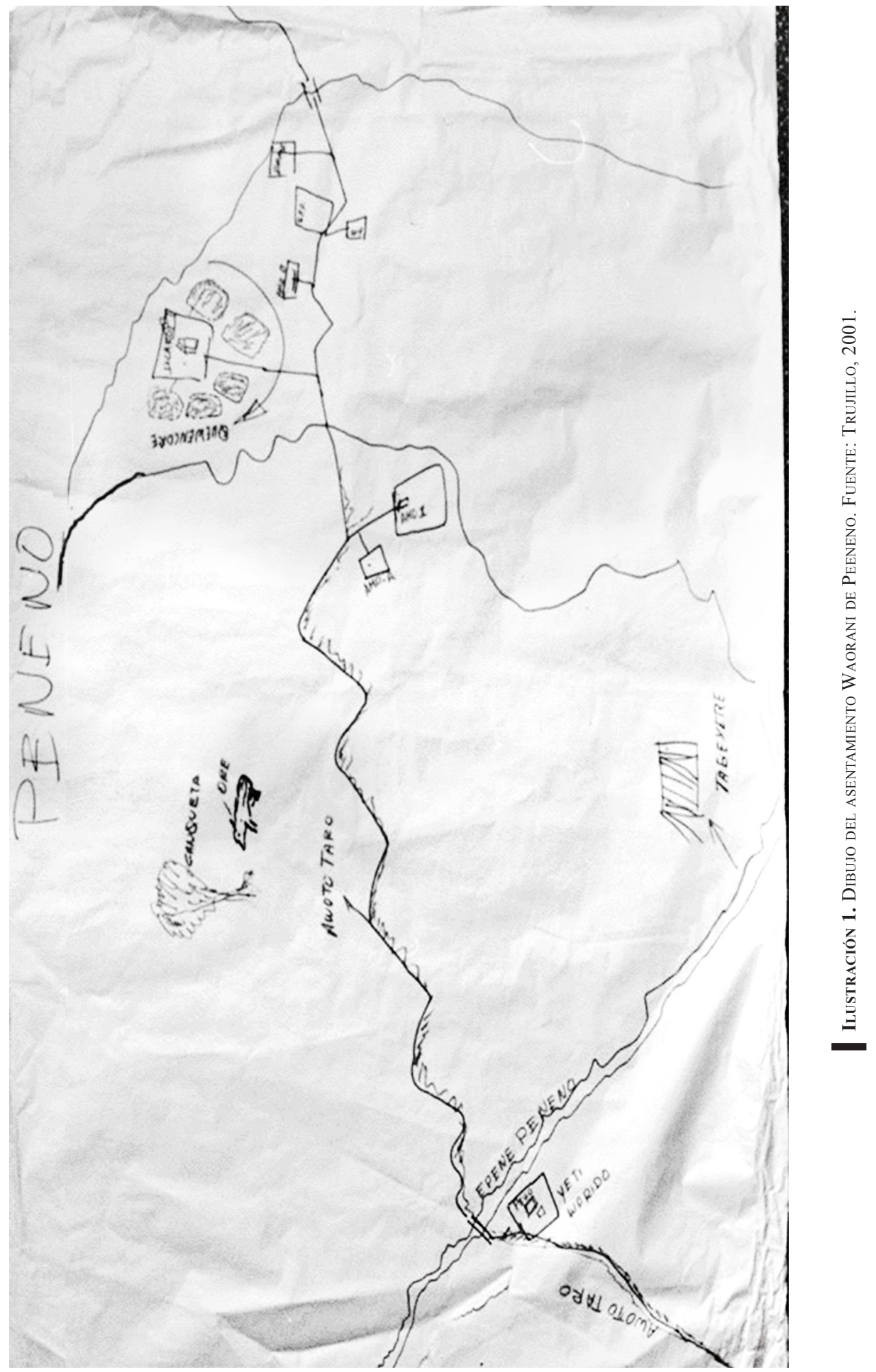




\begin{abstract}
"Davo relata cómo participó en la matanza del 2003 a una familia aislada Tagueiri en la zona de río Menkaro. Nos cuenta que Babe y sus hijos los invitaron, debían vengar la muerte del sobrino de Babe, Carlos ocurrido años atrás. La invitación a una correría es algo que un guerrero no puede rechazar, so pena de ser señalado como cobarde dentro de su grupo y por lo tanto estigmatizado y sancionado socialmente. Davo recuerda que cada guerreo recibió dos cajas de balas, se armaron también con lanzas, pero dice que fueron pocos las que llevaron, caminaron dos días, durmieron en la selva esperando el momento del ataque. Recuerda que en un día muy lluvioso rodearon la casa, les gritaron quienes son, que mujeres les contestaron que no les maten que son sus enemigos. Davo recuerda que dispararon muchas balas, que mataron mucha gente, que el hijo de Babe para decir que si mataron Tagueiri corto la cabeza de uno y lo llevo en una funda, a los que sobrevivieron a las balas les clavaron lanzas, luego cogieron cosas de los Tagueiri y se volvieron, recuerda finalmente que fueron perseguidos por varios guerreros hasta el río Tiwino, que les lanzaron lanzas que prometieron vengarse" (Diario de campo, 2003).
\end{abstract}

Un segundo grupo sería el denominado Wiñaetairi y se localizaría en una amplia zona hacia el este del río Ñashiño, Cononaco en dirección hacia el río Curaray. Davo dice que ha escuchado que son "muchos, con muchas lanzas". Finalmente, un tercer grupo denominado como Iwane localizado en la zona de alto Yasuní del que no se conoce mucho ${ }^{26}$. En la actualidad, luego del fracaso y desencanto que resultó para muchas familias Waorani la vida en el protectorado, muchas familias, retomaron los sitios donde sus padres o ellos nacieron, practicando la vida en la selva, que implica un aislamiento voluntario y una alta movilidad. Ejemplos como Gaba en Ñashiño, Mega en Iro, Oña y Tokari, Karulle y Meñewa son grupos familiares con altísima movilidad, quienes controlan amplios espacios de territorio y son fronteras con las familias aisladas, manteniendo diversos niveles de relación socio-cultural.

\title{
El mito del no contacto
}

Desde 2003 se ha evidenciado, con mayor frecuencia, el número de encuentros violentos entre PIA y sus vecinos étnicos, lo que los ha puesto en complejos escenarios de vulnerabilidad (Cabodevilla, 2013). No existen estudios profundos, ni sistemáticos sobre los PIA en Ecuador y este desconocimiento ha generado una serie de especulaciones sobre estos grupos.

"Kay es hijo de Nampawe, del grupo del alto Yasuní y de quienes se conoce por el contacto realizado
por la misión capuchina y las empresas petroleras, viven ahora en un asentamiento permanente, en
Kawymeno (río de Loras) al que se lo conocía como Garzacocha, un asentamiento nucleado que
aún conserva casas al estilo tradicional Waorani, construidas con hoja de palma y con visibles
entrada y salidas, para huir de los enemigos, los guerreros guardan sus lanzas entre la estructura de
la casa, y el número de fogones y hamacas señala el número de familias que comparten la vivienda,
los Wao son familias ampliadas, unidas bajo complejas alianzas parentales. En la noche la selva
se transforma, una característica bulla inunda el ambiente, miles de insectos que zumban, pero ese
monótono sonido de insectos se rompe con el canto de las mujeres Wao que inunda la selva con un
llamado a sus recuerdos. Kay afirma que los Taromengas y Taromenani son el mismo grupo, con
el cual las familias del Yasuní y de Cononaco-Baameno, mantienen un estrecho contacto, viven
en un gran territorio, se movilizan rápidamente y están por dos o tres meses en sitios donde tienen
sembríos de yuca y saben que hay mucha cacería, allá por el río Nashiño. Me dice que su mujer
Wiñaykamo es Taromenga, de esa zona del Nashiño, que se visitan y cuando lo hacen hay fiesta
mucha carne de ure (wangana) ahumado que llevan los Taromenani, mientras que ellos llevan
mucha yuca y platano" (Diario de campo, septiembre 2002).

El mito del no contacto ha descontextualizado características de las familias aisladas como parte de culturas amazónicas y su relación vital de intercambio, tanto con su entorno, como con sus vecinos (familias waorani, industria petrolera, madereros, etc.). No existen grupos sin 
contacto, a los PIA, no se les podría catalogar como pueblos ocultos ni menos aún sin contacto (Cabodevilla, 2004), puesto que existen evidencias ciertas de sus relaciones, tanto de parentesco, como alianzas simbólicas con otros grupos clánicos Waorani, tales como los Baiwairis, Kempereiris y Kairis, tres de las familias que controlan y habitan, precisamente los territorios del Parque Nacional Yasuní y específicamente las zonas de los ríos Cononaco, Nashiño y Yasuní por donde existen evidencias que se movilizan los grupos aislados (Cabodevilla, 2013; Trujillo, 2011, 2016). El contacto con familias waorani se lo interpreta en varios niveles:

a.) intercambio de productos del bosque, por ejemplo cacería por yuca y plátano o artesanías, por productos de la ciudad; machetes, hachas, sal, ollas, etc.;

b.) simbólicos, los informantes advierten de la existencia de intercambios en fiestas por buena cacería, potenciales alianzas y matrimonio (intercambio libidinal);

c.) guerra producto de venganzas, como forma de consolidar alianzas y reciprocidad parental.

\section{Conclusiones}

La guerra fue una de las principales formas de reproducción cultural para muchos grupos amazónicos (Trujillo, 2011). Para los waorani, la guerra entrelazaba complejos sistemas de alianzas y lealtades grupales, acelerando un intercambio de varios recursos, sean estos materiales o simbólicos. La guerra, fue la institución social que resolvía los conflictos y dinamizaba formas culturales, como el intercambio intra y extra clánico: entre nanikaiboiris (mismos clanes), waranis (otros clanes Waorani) y cuwuris (extraños, no Waorani) (Ibíd., 2006, 2011). En la memoria oral de varios Pikenani entrevistados, la guerra y sus recuerdos los traslada a otros espacios temporales $^{27}$, donde existía una especie de código $^{28}$ mediante el cual justificaban el hecho de la guerra, los asesinatos y sobre todo la venganza (Ibíd., 2011, 2016):

- Cuando un miembro del clan es asesinado por familiares o por enemigos

- Cuando un pariente no regresa de cacería.

- Cuando un brujo de otro grupo es culpable de matar a cualquier miembro de la familia.

Cuando enemigos o extraños ingresan a sus territorios de cacería o recolección.Las familias waorani de los grupos Niwairis (Davo, Pegonta), Waneiris (Kemperi, Iniwa, Pego) y Baiwairis ${ }^{29}$ (Karulle, Kawuya, Awa, Omayewe, Meñewa) que habitan en la ribera del río Shiripuno (Keweriono) hacia el río Cononaco (Baanemo) mantienen contacto con las familias Tagueiri- Taromenani, intercambio que se da en varios niveles, tanto materiales (productos de caza por productos de chacra y recolección), como simbólicos vinculados a guerra, fiestas y potenciales intercambios de mujeres. Karulle, relata haberlos visto "cogiendo huevos de Charapa", sabe dónde están sus casas, algunas habitadas y otras abandonadas, chacras (kewenkores) y caminos de cacería desconocidos (Tarome). Los grupos Niwairis y Babeiris, son enemigos de los PIA, y su relación social se caracteriza por tiempos de paz y tiempos de guerra y venganzas ${ }^{30}$ (Ibíd., 2006, 2011, 2016).

27 El derecho natural a usar sus lanzas se basa en la venganza, puesto que la muerte de un familiar debe ser vengada como un imperativo, nuestros entrevistados afirmaron que existe miembros de un clan, niños inclusive que esperan ser adultos para vengar la muerte de sus familiares "y poder vivir tranquilos".

28 Denominado por mis informantes como código guerrero.

29 Relato de Wane Kawuya (2013). Relato de Davo, Babe, Yety, Karuwe y Omayewe (2010). Relato de Kemperi y Awa 2010.

30 Para Davo, la venganza y muerte por lanzas son eventos relacionados a muertes de familiares por brujerías enviadas por enemigos. Muchas de las guerras interclánicas fueron producto de supuestas muertes ocasionadas por brujos o hechiceros, quienes utilizaban sus poderes mágicos para eliminar a sus enemigos. La muerte con lanzas de Ompure ocurrida en marzo del 2013, se debería a que Ompure era conocido como "Iro" o "shaman-brujo" por lo que miembros enfermos de una familia de aislados habrían ido para que los trate, la enfermedad y posterior muerte de miembros de este clan que cuando no es por lanzas o accidentes naturales es relacionado a eventos mágicos, habría provocado la venganza acusándolo de haber matado a los enfermos (Entrevistas, 2013). 
"El Shiripuno, Keweriono en lengua Wao, es un largo río que desde su cabecera aguas arriba, une y atraviesa varios asentamientos Waorani hoy convertidas en comunidades. Dicen es el río de los caníbales (cowuris), Awa Baiwa aún recordaba que sus abuelos le contaron que por este río subían los caucheros a llevar Waorani, los que nunca regresaron. Desde el puente casi 6 horas río abajo se une con el Cononaco Chico formando una Y, allí está el antiguo campamento de la CGG, la compañía francesa de sísmica que estuvo en esta zona en la década de los 80 del siglo pasado, aún se pueden observar los pilotes de las casas del campamento. Allí vive Gankimo Karulle, pertenece al grupo Bawairi, es hijo de Yate, hermano de Wane, padre de Kemperi, por lo que tiene parentesco con el grupo de Baameno. La casa de Karulle es la frontera más cercana entre los Tagueiri. Karulle vive de forma tradicional, lo que significa habitar una casa construida de hoja de chonta, vivir de la cacería, recolección de frutos de monto y de la pesca que es abundante en el Keweriono. El viaje desde el punto conocido como el puente del Shiripuno en la vía Auca, hasta la casa de Karulle toma 8 horas entre un sinuoso río y la exuberante vegetación, es el mes de noviembre y el río está bajo mostrando grades playas donde desovan las tortugas (charapas), sus huevos son muy apetecidos por su sabor entre todas las culturas amazónicas. Llegamos a la noche a la casa de Karulle, podemos ingresar porque nuestro amigo Enkeri, hijo de Minkaye, quien está casado con Cahuo del grupo familiar de Baiwa, caso contrario sería imposible el ingreso, puesto que el grupo de Enkeri que pertenece a los denominados "del río arriba" son enemigos, únicamente esta alianza matrimonial permitió el acceso a estas zonas. Karulle y su familia reposaban alrededor del fogón en hamacas, eran 5 personas tres adultos y dos niños. Varios papagayos dormían en la parte superior de la casa. Menga, quien vivía en la vía Maxus, cerca de los pozos petroleros Iro, era otro guerrero Waorani, del mismo clan que Karulle me había contado años atrás que tanto papagayos como el águila arpía, los tienen como mascotas porque alertan cuando se acercan los enemigos. En la madrugada los papagayos chillaron alertando a Karulle quien salió rápidamente con su lanza, ruido de muchos pasos, gritos y conversaciones durante un buen tiempo hasta antes de un silencio que ya anunciaba el amanecer. Karulle, continuaba en su hamaca cerca al fuego mirando y oliendo varias patas de huangana ahumadas que la noche anterior no estaban, no las comía solo las olía, y las miraba de todos los lados, tampoco intento convidarnos. Salimos temprano rumbo a Baameno, el Shiripuno se une unas horas más con el río Tiwino y forma el Cononaco, ya más grande y navegable, toda esa zona es tierra Tagueiri, ayer me dice Enkeri estuvieron visitando a Karulle, le dejaron patas de ure (huangana) y se llevaron yuca y plátano, le han dicho que estan escondidos por la matanza de abril del 2013, que están con hambre porque no tienen yuca, que los están vigilando, que no se asuste, que volverán" (Diario de campo, noviembre 2013).

La declaratoria de la ZITT (Papalardo, 2013), que abarca parte de la territorialidad de los PIA, en cierta forma ha reconfigurado, las relaciones con los actores externos, especialmente madereros, con quienes los PIA han mantenido encuentros violentos desde 2001. Sin embargo y a pesar de la declarada protección y auspicio por las entidades del Estado, estas en realidad son impotentes ante la actual situación de vulnerabilidad de estos grupos, cada día más cercados. En especial, la actual situación de las familias Tagueiri-Taromenani de la zona Tivacuno-Cononaco Chico (Peeneno-Baameno)- Tiwini-Cuchiyaku (Menkaro) es crítica, debido al asedio sistemático que han sufrido, por los clanes Waorani enemigos, por los clanes Waorani aliados y por los agentes externos (ampliación de la frontera agrícola, extractiva, urbana).

¿Cuál es el futuro de estas familias de indígenas aisladas? Luego de la última matanza en abril del 2013, su situación es más compleja aún, sus espacios de movilidad se reducen cada vez más y la relación de vulnerabilidad y conflictividad resultante de una reducción de espacio (territorio) y del potencial tiempo, para un encuentro con enemigos, es cada vez más corta. Un nuevo encuentro violento con familias waorani enemigas, potencialmente los eliminaría por completo, su extinción física es una probabilidad cierta. Por otro lado, concluimos, que una lenta pero sostenida fisión entre las familias Tagueiri-Taromenane y los clanes de Kairi, Kemperiri y Baiwairi, es también una posibilidad. El intercambio libidinal ha sido una de las más eficientes estrategias de supervivencia de los grupos guerreros amazónicos. Quizá eso es lo que está sucediendo en la zona intangible, en las amplias selvas del Kewerioro, Baameno, Nashiño, Kawymeno y Curaray. 


\section{Bibliografía}

Cabodevilla, Miguel Ángel. 2013, Una tragedia ocultada, CICAME, Quito.

2004, El exterminio de los pueblos ocultos, CICAME, Quito.

FLACSO-Fundación Pachamama. 2012, "Situación del grupo Armadillo- Cononaco -Chico- vía Tigüino", Informe borrador, Quito.

Ministerio de Ambiente. 2012, "Plan de medidas cautelares para la protección de los pueblos indígenas en aislamiento Tagaeri-Taromenani. (PMC-PIAs), Actualización de los "Criterios Técnicos Para la Evaluación de Planes de Aprovechamiento Forestal, Ocupación Territorial, u Otros Planes de Manejo en Áreas Colindantes o Cercanas a los Territorios Tagaeri-Taromenane en las Provincias de Orellana y Pastaza", Presentación a la Fiscalía de Orellana, Ministerio de Justicia, Coca.

Narváez, Roberto. 2016, Quiénes son los pueblos en aislamiento en el Yasuní, Documento de trabajo.

Ministerio de Justicia Derechos Humanos y cultos. 2007, "Plan de Medidas Cautelares para la protección de los Taromenani y Tagaeri", 13 de julio de 2007. Quito, Ecuador .

Presidencia de la República. 2007, "Política nacional de los pueblos en situación de aislamiento voluntario", documento de consulta, Quito.

Rivas, Alexis et al. 2014, Consultoría para la elaboración de las políticas públicas de los Pueblos Indígenas en Aislamiento. Secretaría de gestión de la Política.

Rivas, Alexis. 2003, "Sistema mundial y pueblos indígenas en la Amazonía. A propósito del ataque a los Tagaeri”, en: revista ICONOS (17), Facultad Latinoamérica de Ciencias Sociales-FLACSO,Quito-Ecuador, pp. 21-31.

Pappalardo, Salvatore Eugenio et al. 2013, Uncontacted Waorani in the Yasun'' Biosphere Reserve: Geographical Validation of the Zona Intangible Tagaeri Taromenane (ZITT).

Trujillo, Patricio. 2016, Código Guerrero y encuentro con lanzas. FIAAM, en prensa, Quito.

2011, Bito cowuri, Boto Waorani, la fascinante historia de los Waorani FIAAM, Quito.

2006, "The fascinating story of the Huaorani or "the true humans". Acta Americana. Vol 14, N. 1, Journal of the Swedish Americanist Society.

2001, Salvajes, civilizados y civilizadores; la Amazonía ecuatoriana, el espacio de las ilusiones, Fundación de Investigaciones Andino Amazónicas (FIAAM)- Abya Yala, Quito. 